\title{
Hands on Particle Physics
}

\section{Christine Sutton*}

Address

Nuclear and Astrophysics Laboratory, University of Oxford, Keble Road, Oxford OX1

3RH, UK

E-mail: ${ }_{1}^{\mathrm{c}}$ sutton 1 Qphysics.ox.ac.uk

ABSTRACT: This presentation describes a project to create a national resource of demonstrations and displays related to high energy particle physics, which can be used by the UK particle physics community in public lectures, open days, exhibitions and similar events. It contains high quality display boards, hands-on apparatus and multimedia.

\section{Introduction}

There is significant interest in high energy particle physics among the public and schools in the UK. However, with no national facility dedicated to particle physics, it is difficult for the public to see particle physics in action. For this reason a consortium of particle physicists set up the Particle Physics Exhibits Project (PPEP), with funding from the Particle Physics and Astronomy Research Council (PPARC).

\section{What is PPEP?}

PPEP is a library of demonstrations, interactive models and displays for particle physicists throughout the UK to use in taking particle physics to the public. It is designed to enable the interactive presentation of particle physics at all kinds of public event, from demonstration lectures at schools to full-scale exhibitions. The exhibits are not designed for use in unattended exhibitions, and are not self-explanatory. The emphasis instead is on providing attractive displays and apparatus that enable particle physicists to engage with an audience - to give them "something to talk about". Now complete, PPEP has been used in major public events such as the Edinburgh International Science Festival, as well as at more local events such as Particle Physics Masterclasses for schools.

\footnotetext{
${ }^{*}$ Speaker.
} 
For the purposes of PPEP, we have divided particle physics into 12 topics, each of which is represented by a set of professionally designed display panels and various demonstrations. Each set of display panels consists of four linked graphics panels, which are folded easily into a bag for carrying. The demonstrations include models, interactive apparatus, and interactive computer programs. Some of these were existing items, but others have been specially designed and built for PPEP. The apparatus varies in size from items that are simply transportable by a single person, to others that are large and heavy. Any number of items may be borrowed, from a single set of display boards or piece of apparatus, to the complete collection, for which a small lorry is required for transport.

Further information about all the exhibits can be found at: http://hepweb.rl.ac.uk/ppep/.

\section{The twelve topics}

The topics were chosen to cover the fundamental ideas of particle physics (particles, forces, and the structure of matter), the main experimental techniques (detectors, accelerators, and major laboratories), as well as aspects more closely related to particle physics in the Universe (neutrinos, symmetry, and the Big Bang). These topics touch upon all areas of current particle physics research in the UK, including for example cosmic rays, antimatter, dark matter, and the quark-gluon plasma. The following list indicates the twelve topics, and the demonstrations that exist for each of them.

- The particles of matter Real particles are demonstrated with a 12-faced cosmic ray hodoscope, about $1 \mathrm{~m}$ across, in which LEDs light up the tracks between opposite pairs of scintillator. In addition, there is an easily portable, PC-driven apparatus to measure the muon's lifetime.

- The forces of nature Demonstrations show the particle and wave nature of the electron, with items based on commercially available electron $\mathrm{e} / \mathrm{m}$ and diffraction apparatus. A rubber "spacetime sheet" provides an analogy with the distortion of space in the region of a massive body.

- The structure of matter This includes a working modern replica of Rutherford's scattering experiment with an alpha source and gold foil. There is also a "billiardtable" analogue in which collisions of billiard balls set up by the user are recorded by a video camera, so that time-lapse replays can show "particle tracks".

- Particle accelerators Two demonstrations invite you to "drive your own accelerator". One is an adapted e/m apparatus which allows the user to see how beam energy (electric field) and magnetic field affect the size of a circular electron beam. The other is a console with a display screen, which allows the operator to fill, accelerate and collide beams at LEP. (A web-based version of the simulator can be found via: http://hepweb.rl.ac.uk/ppep/exhibits/accels/CernComplex/LEPsim.htm 
- The LHC The models here are a 1:50 scale model of ATLAS, which can be taken apart to show different components, and a simple magnetic analogue of the Higgs field.

- LEP Interactive web-based PC software shows events from LEP which can be rotated and zoomed. A model with fluorescent wires shows one of the events in $3-\mathrm{D}$, which can be rotated just as on the PC.

- HERA At present there are display boards only for HERA, but no specific demonstrations.

- Particle physics world-wide For this topic the display panels are made interactive with LEDs that show the locations of various laboratories and experiments around the world.

- Particle detectors The display panels here include actual examples of detectors - scintillator, phototube, wire chambers, silicon detectors, and a prototype trigger board for ATLAS. In addition, there is an exhibit with a UV-illuminated scintillator and wavelength-shifter, and several enlarged bubble chamber events on acetate sheets to view on a lightbox.

- The mysterious neutrino A coupled pendulum illustrates neutrino oscillations, and an interactive software package follows neutrinos from the appropriate source to interactions in the SNO, SOUDAN, and MINOS detectors.

- Symmetry in the Universe The display boards contain a simple illustration of parity and time reversal with two working clocks - one normal and one running backwards - and a double-sided mirror.

- Matter in the Universe A scale model shows the Boulby Mine in North Yorkshire, and the location of the dark matter detectors below ground. There is also a fullscale model of a dark matter detector module, as used by the UK Dark Matter Collaboration

\section{The PPEP multimedia}

This is a professionally-produced interactive multimedia version of the display boards, with animations to bring the topics to life. This can be used in either in stand-alone mode at an exhibition, or in a web browser (see http://hepweb.rl.ac.uk/ppep/multimedia.htm). In addition there is a short self-looping presentation, with a selection of pages from the full multimedia display. For the purposes of the multimedia the topics are grouped into four themes:

- Building the Universe: Particles, Forces, and the Structure of Matter

- How to see Particles: Detectors, Accelerators, and Particle Physics World-Wide 
- The Big Machines: LEP, HERA, and the LHC

- Particles and the Big Bang: Neutrinos, Symmetry, and Cosmology

\section{Use of the exhibits}

The PPEP exhibits are available for loan to members of the UK particle physics community. The items are normally stored mainly at the Rutherford Appleton Laboratory, although they return to the originating institute for repair and refurbishment. A set of web pages gives details of all items available for loan, including instructions for operation and transportation, and safety requirements. Applications to borrow items are processed via a web-based booking system, which is moderated by one of the PPEP team members. It is the responsibility of the person booking the items to arrange appropriate transport. However an additional award from PPARC has made subsidies possible, eg for van hire.

To date (November 2001) there have been more than 120 bookings, varying from one or two items for a schools' talk, to the complete collection for major exhibitions. Items have appeared at the Edinburgh International Science Festival in 2000 and 2001, at the British Association's Festival of Science in 2000, at the Royal Society's New Frontiers in Science Exhibition (2000 and 2001) and at the Association of Science Education's Annual Meeting in 2000 (and again in 2002). (Some photographs at these exhibitions can be seen at http://hepweb.rl.ac.uk/ppep/OnShow/exhibitions.htm)

\section{Acknowledgments}

The Particle Physics Exhibits Project was made possible through an award of £79,760 from the Particle Physics and Astronomy Research Council (PPARC), under the National Award Scheme for the Public Understanding of Science and Technology. We are also grateful for additional support from the Physics Departments of the participating institutions - the Universities of Birmingham, Bristol, Edinburgh, Oxford, University College London, and the Rutherford Appleton Laboratory. Valuable contributions have also been made by Royal Holloway, University of London, and Queen Mary, University of London. PPARC has made a second award to the project to cover the costs of maintenance and to allow a "travel fund" to subsidise the costs of transporting items. 